\title{
Arrhythmogenic Right Ventricular Dysplasia - Value Of Fat Suppresion In MRI And Black Bold Spin Echo Images
}

\author{
AK SHARMA, S SINGH
}

Ind J Radiol Imag 2006 16:2:233-234

Key words : ARVD, Cardiac MRI, fatty infiltration, myocardium

\section{Introduction}

ARVD is a poorly understood and often inadequately diagnosed form of cardiomyopathy. Cardiomyopathy leads to damage of the myocardial walls of the heart, causing them to become thickened and chambers to enlarge. Patients with ARVD often have arrhythmias, which can increase the risk of sudden cardiac arrest or death.MRI can be confirmatory by its unique ability to identify and characterize fatty tissue infiltration in right ventricular free wall. Other MR criteria are severe RV dilation, localized $\mathrm{RV}$ aneurysms, mild global dilatation and regional RV hyperkinesias.ECG findings are Epsilon wave just beyond the QRS complex in lead V1, wide QRS complex duration of greater than 110 msec in leadV1, inverted T waves in V1 V3, late potentials in LBBB.VES

\section{Case report}

A 39 year-old came to hospital with episodes of ventricular arrhythmias and also had a previous history of cardiac arrest .On admission ECG shows VT at a rate of $180 / \mathrm{min}$ with LAD with LBBB.Precordial concordance positive. Resting ECG showed T inversion in V1-V6. Holter showed frequent bigemini. No evidence of NSVT/VT or sinus pauses. Coronary study was normal with EF of $60 \%$.Echocardiography did not reveal any abnormality. On these findings (Clinical/ECG), diagnosis of Right ventricular Dysplasia was made.

Cardiac MR was performed on $1.5 \mathrm{~T}$ unit using synergy coil and the following sequences. BFE axial sequence was obtained to to show the anatomic detail of cardiac chambers(Fig1). Axial T1-weighted black blood which showed evidence of fibro fatty replacement of myocardium of right ventricular free wall, mild RV dilatation, mild global dilatation and regional RV hypokinesia(Fig2).RVOT was also dilated (Fig. 4). Fat suppression sequence was also done, which confirmed fatty infiltration of right ventricular wall (Fig 3).

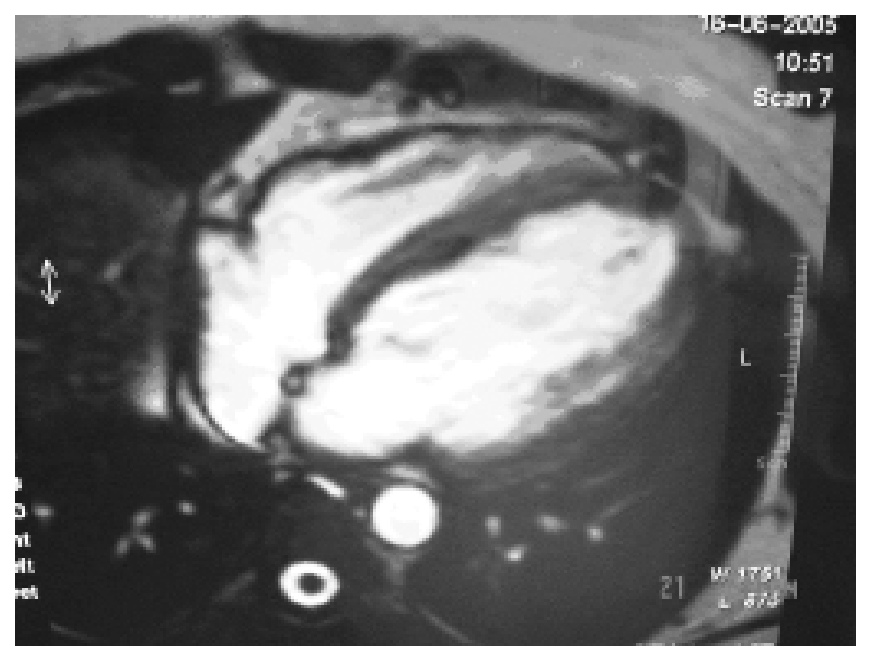

Fig.1: BFE sequence showing anatomical detail of all four chambers.

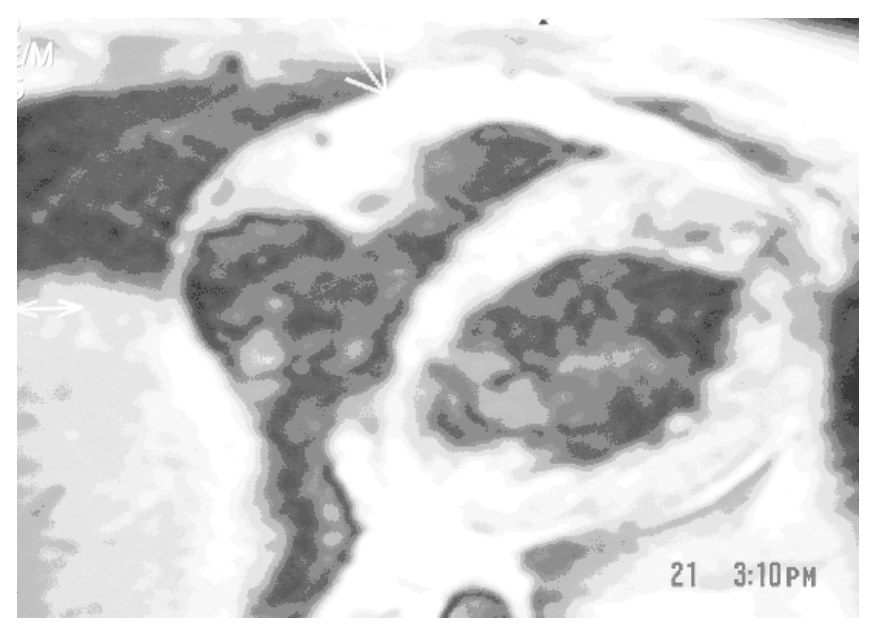

Fig.2: Black blood sequence showing infiltration of fat in myocardium of right ventricle as hyper intense areas.

From the Department Of Radiology And Imaging Gb Pant Superspeciality, Hospital And Mam College, New Delhi -2 (Delhi University)

Request for Reprints: Department Of Radiology And Imaging Gb Pant Superspeciality, Hospital And Mam College, New Delhi -2 (Delhi University) 
234 AK Sharma et al

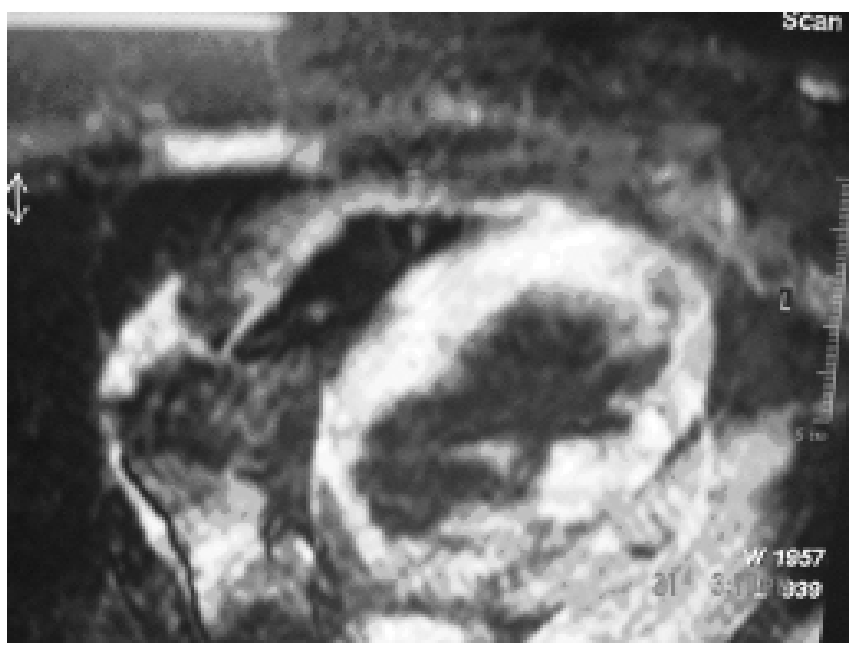

Fig. 3: Fat suppressed black blood image showing suppression of fat in myocardium of right ventricle.

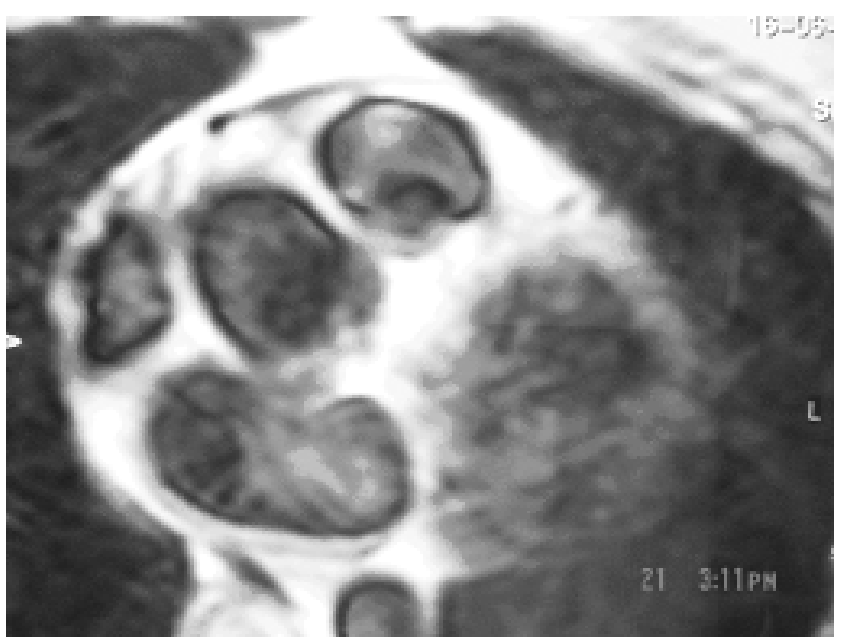

Fig 4 Black blood sequence showing dilated outflow tract

\section{Discussion}

ARVD is being diagnosed with increasing frequency, mostly because MR allows improved recognition of myocardial fatty and fibro fatty replacement (1).Several studies have reported on the use of MR imaging to detect the characteristic high signal intensity of fat in the right ventricular myocardium on T1 weighted images. However,recent studies demonstrated that significant fatty infiltration of right ventricle occurs in more than $50 \%$ of normal hearts in elderly. Mehta et al found signs of fatty replacement in only $22 \%$ of 27 patients with ventricular tachycardia with LBBB.Menghetti etal reported sensitivity and specificity of $67 \%$ and $100 \%$.respectively, with use of spin-echo MR imaging.

Several studies examined the value of using MR imaging in patients for whom the first manifestation of right ventricular disease was right ventricular outflow tract tachycardia.

This is associated with focal structural and wall motion
IJRI, 16:2, May 2006

abnormalities of the ventricular outflow tract and that structural abnormalities observed with MR imaging were often not detected with echocardiography.MR imaging can also be used to assess both systolic and diastolic function in great detail. Several studies have addressed the presence of right ventricular diastolic dysfunction as an early marker of disease; even systolic function is still preserved.

The typical criteria that can be demonstrated with MR imaging are Fatty infiltration of the right ventricular myocardium with high signal intensity on $\mathrm{T} 1$ weighted images, fibro fatty replacement, which leads to diffuse thinning of the right ventricular myocardium, aneurysms of the right ventricle and right ventricular outflow tract. Dilatation of right ventricle and RVOT, regional contraction abnormalities and global systolic dysfunction. Castillo $E$ etal suggested that intramyocardial fat detection in ARVD was better with fast SE MR imaging alone and combined with fat suppression than was gated SE MR imaging in vivo.Abbara $S$ et al used fat suppressed in addition to non fat suppressed and observed that interobser agreement and confidence in diagnosis and evaluation of intramyocardial fatty infiltration has increased. We have also used same pattern in presented case.

\section{REFERENCES}

1 Castillo E et al Arrhythmogenic Right Ventricular Dysplasia: Ex Vivo and in Vivo fat detection with BlackBlood MR Imaging.Radiology,July,2004;232(1):38-48.

2 S.Abbara, R.Q.Migrino, et al. Vale of fat suppression in the MRI evaluation of suspected Arrhythmogenic right ventricular Dysplasia. Am.J.Roentgenol; March1, 2004; 182(3):587-591.

3 R.R Edelman .Contrast -enhanced MR imaging of the heart: Overview of the literature.Radiology, September 1 , 2004; 232(3):653-668. 